\title{
Das Haus Elis und das Haus Davids: Wie Gott sein Wort zurücknehmen kann
}

\author{
James Alfred Loader \\ Institut für Alttestamentliche Wissenschaft und Biblische Archäologie \\ Evangelisch-Theologische Fakultät, Universität Wien \\ Professor extraordinarius, Unisa, S A
}

\begin{abstract}
The house of Eli and the house of David: how God can go back on God's word

The interpretation of the rejection of Abiathar in terms of the Eli tradition offers an important indication of the intention of the Succession Narrative. The work does not only have a positive as well as a negative tendency as far as the Davidic dynasty is concerned, but the relationship between these two sides is one of conditionality. Therefore the critical aspect of the Succession Narrative contains both acceptance and warning. A sketch of the broader theological framework is given in which the interplay of God's love and his demand of obedience is understood in its historical context, including the redactional perspective.
\end{abstract}

\section{EINLEITUNG}

Das Motiv in den Geschichten vom Priester Eli und vom König David, das dem religionspädagogischen Interessengebiet des verehrten Kollegen Gottfried Adam am nähesten kommt, ist das Motiv des erziehenden Vaters. Die beiden Figuren, die diesen Gedanken in der Geschichte von der Thronnachfolge Davids vergegenwärtigen, sind besondere Personen, weil die eine ein Priester, und die andere ein König ist. Beide sind negative Folien des idealen Erziehers, weil keiner von beiden seine Söhne mit der angebrachten Disziplin erziehen kann. Zwar handelt es sich um ein negatives Motiv, aber dennoch um ein religiöses sowie pädagogisches. $\mathrm{Da}$ - wie z.B. im Proverbienbuch zu beobachten ist - negative Vorbildern eine durchaus positive erzieherische Wirkung 
zugeschrieben werden kann, möchte ich dem Jubilar folgende Überlegungen zu diesem Aspekt der Geschichtsdeutung des Deuteronomistischen Geschichtswerks in kollegialer Verbundenheit widmen.

\section{DIE BEIDEN ASPEKTE DER ERZÄHLUNGSTENDENZ}

Seit der klassischen Arbeit Leonhard Rosts', hat man die Tendenz der Geschichte von der Thronnachfolge Davids meist als pro-davidisch bzw. pro-dynastisch ${ }^{2}$ oder dynastiekritisch $^{3}$ oder als Kombination der beiden ${ }^{4}$ gedeutet. lch würde argumentieren, daß weder die Bezeichnung einer anti-dynastischen noch die einer pro-dynastischen Tendenz angebracht ist und ebensowenig die Vorstellung einer Kombination der beiden. Eine andere Deutung ist möglich, nach der man vielmehr von den kritischen bzw. positive Aspekten des literarischen Werkes reden kann.

Einerseits ist die kritische Tendenz der Erzählung kaum zu übersehen ${ }^{5}$. David ist ein unfähiger König, Ehebrecher, Mörder ${ }^{6}$ und als Vater seiner erzicherischen Aufgabe keineswegs gewachsen ${ }^{7}$. Er läßt seinen Söhnen entweder mit Erlaubnis ${ }^{8}$ oder durch

\footnotetext{
' Die Überlieferung von der Thronnachfolge Davids, Stuttgart 1926.

2 Rost, a.a.O., G. Von Rad, Der Anfang der Geschichtsschreibung im alten Israel (1944), in Gesammelte Studien zum Alten Testament, München 1965; F. Mildenberger, Die vordeuteronomische Saul-DavidÜberlieferung, Diss., Tübingen 1962; R.N. Whybray, The Succession Narrative. A study of II Sam. 9-20 and I Kings 1 and 2, London 1968; H.-J. Hermisson, Weisheit und Geschichte, in H.W. Wolff (Hrsg.), Probleme biblischer Theologie. Gerhard von Rad zum 70. Geburtstag, München 1971; T. Mettinger, King and Messiah. The civil and sacred legitimation of the Israelite kings, Lund 1976; P.K. McCarter, Plots, true or false. The Succession Narrative as court apologetic, Int. 35 (1981), S. 355-367.

${ }^{3}$ L. Delekat, Tendenz und Theologie der David-Salomo-Erzählungen, in F. Maass (Hrsg.), Das ferne und das nahe Wort, Berlin 1967.

4 E. Würthwein, Die Erzählung von der Thronfolge Davids. Theologische oder politische Geschichtsschreibung? Zürich 1974: Eine kritische Schicht sei um eine positive Schicht erweitert worden. T. Veijola, Die ewige Dynastie. David und die Entstehung seiner Dynastie nach der deuteronomistischen Darstellung, Helsinki 1975: Spätere redaktione!le Tätigkeit sei für die unterschiedlichen Pro- und Anti-Elemente verantwortlich.
}

5 Vgl. J.A. Loader, Jedidiah or: Amadeus. Thoughts on the Succession Narrative and wisdom, OTWSA $27 / 28(1986), 187 \mathrm{ff}$.

${ }^{6} \mathrm{Vgl}$. die Bathseba-Episode, 2. Sam. 11, und die Kritik Nathans, 2. Sam. 12.

${ }^{7}$ Vgl. 2. Sam. 13,21 (Amnon), 1. Kön. 1,6 (Adonia); Prv. 13,24; 17,25.

${ }^{8}$ Z.B. 2. Sam. $14,21.33 ; 15,19$. 
Unkenntnis ${ }^{9}$ Abenteuer zu, die in Katastrophen ausarten. Auch sein Nachfolger, Salomo, zeigt moralische Schwächen auf, indem er die Versicherung, die er seiner Mutter einmal gegeben hat, mißachtet, fadenscheinige Gründe erfindet, um seine Gegner ermorden zu können, und sogar das Heiligtum entheiligen läßt ${ }^{10}$. Des Vaters abwechselnde Lethargie und Impulsivität kostet dem Sohn beinahe den Thron ${ }^{11}$. Also ist der These Rosts, daß die Erzählung ad majorem gloriam Salomonis geschrieben worden sei ${ }^{12}$, ebensowenig aufrecht zu halten wie die oben erwähnten "pro-dynastischen" Interpretationen.

Auf der anderen Seite ist diesen Exegeten ein gewisses Recht auch nicht abzusprechen. Hat David doch tatsächlich die Schwierigkeiten, von denen so ausführlich erzählt wird, überstanden und hat Salomo doch die Turbulenzen zu seinem Gunsten überwunden. Am Ende der Geschichte setzt der Verfasser ein klares Signal, daß die Erzählung (auch) so zu lesen ist: Es heißt, daß das Königtum Salomos trotz allem gefestigt wurde (1. Kön. 2,46). Also ist nicht zu übersehen, daß die davidische Dynastie letztendlich doch etabliert worden war und daß der positive Erfolg dem negative Faktor überlegen war. Neben dem kritischen entwickelt sich daher ein positive Faktor.

Es kommen vier deutliche Hinweise in der Geschichte vor, "in denen der Verfasser in Gestalt eines positiven theologischen Urteils sich über Gott und sein Verhältnis zu den geschilderten Ereignissen ausspricht"13.

In 2. Sam. 11,27 heißt es schlicht mit Verweis auf Davids Verhalten in der Bathseba-Uria-Geschichte, daß, "was David getan hatte, Jahwe mißfiel”; und im direkt folgenden Vers heißt' es, daß das der Grund für die Bestrafung des Königs durch Jahwe unter Mitwirkung des Propheten Nathan war. Von Rad hat in dem Sinne Recht, daß

9.B. 2. Sam. 13,13.21; 1. Kön. 1,18.

${ }^{10}$ Vgl. 1. Kön. 2, wo er sein Wort unverschämt bricht und dem Asylrecht keinerlei Respekt zeigt.

$"$ Vgl. 1. Kön. 1, wo Davids mangelhaftes Wissen um das, was in seinem Königreich vor sich geht, zudem ein pathetisches Bild herstellt. Statt sich nach weisheitlichem Ideal tugendlich beraten zu lassen (Prv. $15,22)$, wird er von Bathseba und Nathan manipuliert.

12 L. Rost, Die Überlieferung von der Thronnachfolge Davids, S. 126.

13 G. von Rad, Der Anfang der Geschichtsschreibung im alten Israel, Gesammelte Studien zum Alten Testament, S. 181-182. Nach ihm sind drei solche Stellen belegt (2. Sam. 11,27; 12,24; 17,14). Ich meine, eine vierte (1. Kön. 2,27), die den Fokuspunkt dieses Artikels darstellt, soll genauer beachtet werden. 
dieses Urteil "positiv" sei, insofern es in eine kategorische Aussage gefaßt wird. Inhaltlich ist es aber durchaus kritisch, also eine negative Beurteilung von Davids Verhalten. Die die Erzählung durchwaltende Kette von irdischen Kausalitäten wird nicht durchbrochen, aber die Folgerung aus dem Theologumenon muß lauten, daß Jahwe hinter den Kulissen wirksam ist.

Im nächsten Kapitel "steht nun das Wort von der ganz irrationalen Liebe Gottes" zum kleinen Prinzen, "womöglich noch isolierter im Zusammenhang"14, und zwar in 2. Sam. 12,24. Im nächsten Vers wird die in die Zukunft weisende Bemerkung durch einen von Von Rad nicht beachteten, ganz nüchternen Bericht über den Namen des Neugeborenen verstärkt. Auch hier ist der Prophet Nathan unter Erwähnung von Jahwe beteiligt. Der Knabe hieß Jedidja - Amadeus - "Geliebter Jahwes". So wird wieder die Parallelität von menschlichem und göttlichem Handeln gezeigt: Nach der geschichtstheologischen Sicht des Erzählers geht der irdische Ablauf der Dinge ohne Wunder und Mirakel vor sich, aber unter der Oberfläche wirkt die göttliche Gegenwart als eine Macht, mit der gerechnet werden muß. Der Erzähler bestätigt das, indem er Adonia, den Verlierer, ein Geständnis in den Mund legt: "Das Königtum war mein ... aber nun hat sich das Königtum gewandt und ist meinem Bruder zuteil geworden; von Jahwe ist es ihm zuteil geworden" (1. Kön. 2,15).

An dem Punkt, der entscheidend für das Fortbestehen von Davids Königtum war, weist der Erzähler zum drittenmal darauf hin, daß der Ablauf der Geschichte von Jahwe gelenkt wurde. Nach 2. Sam. 17,14 war es Jahwe, der es "so gefügt hatte, daß der kluge Rat Ahitofels vereitelt wurde, damit Jahwe Unheil über Absalom bringen konnte". Weil die Kausalitätskette der Ereignisse nur aufweist, daß Absalom einen militärischen Urteilsfehler begangen hat, ist eine klare Bemerkung in diesem Sinne notwendig, um die geschichtstheologische Perspektive des Verfassers an dieser gravierenden Wende zu zeigen.

Aus diesen Gründen sind wir berechtigt zu folgern, daß die Geschichte von der Thronnachfolge Davids, somit das DtrG, nicht nur als politische Tendenzschrift zu lesen ist, sondern vielmehr als Interpretation der (auch politischen) Geschichte, in der eine theologische Tendenz hervorragt. Zwei Aspekte kennzeichnen diese Geschichtstheolo-

\footnotetext{
${ }^{14}$ Von Rad, a.a.O., S. 183.
} 
gie: die menschliche Seite, wo der Mensch in eigener Verantwortung auftritt und auftreten soll, und die göttliche, wo Gott die menschliche Schicksale fügt. Diese beiden Aspekte sind nun weiter aufzuarbeiten, und zwar hinsichtlich eines Motivs, das nicht eine Manifestation des Grundthemas der Geschichte ist, das also nicht direkt die Grundfrage der Erzählung, "wer nach meinem Hern, dem König, auf seinem Thron sitzen wird” (1. Kön. 1,20.27), zu beantworten versucht. Es wird sich zeigen, daß sich auch in einem Nebenthema die zugrundeliegende Geschichtstheologie des Verfassers auswirkt.

\section{DAS HAUS ELIS ALS VORBILD FÜR DAS HAUS DAVIDS}

\section{(a) Der Abjathar-Abschnitt als viertes Theologumenon}

Von den ersten drei perspektivischen Aussagen des Verfassers war die erste (2. Sam. 11,27) dynastiekritisch, die beiden anderen (2. Sam. 12,24; 17,14) waren dem Hause Davids positiv gesonnen. Die nun zu behandelnde vierte (1. Kön. 26-27) steht in der Mitte der Beschreibung der ersten Maßnahmen, die Salomo bei seinem Regierungsantritt unternahm. Beschrieben wird das Auftreten des neuen Königs gegen seine wirkliche oder potentielle Opposition: den Rivalen, Adonia (1. Kön. 11,13-25), dessen Komplizen, Abjathar (Vv. 26-27) und Joab (Vv. 28-34.35), sowie gegen den Benjaminiten, Schimi (Vv. 36-44 ${ }^{15}$ ).

Obwohl es in der ganzen Geschichte von der Thronnachfolge um die davidische Dynastie geht, also um das Haus Davids, spielt ein anderes Haus auch eine wichtige, wenn auch beschränkte Rolle. Wichtig ist das Haus Elis nämlich deshalb, weil seine Erwähnung gerade an einer Stelle vorkommt, wo die theologische Geschichtsdeutung des Verfassers nicht nur ersichtlich, sondern gar thematisiert wird. Als Geschichtsdeutung wird diese Stelle noch deutlicher als sonst gekennzeichnet, weil hier formal auf die aktuelle Vergangenheit Bezug genommen wird. Es handelt sich um 1. Kön. 2,27.

\section{(b) Die Sonderstellung des Abjathar-Abschnittes}

$\mathrm{Da}$ der Abjathar-Abschnitt in dieser Reihe einen betonten Platz hat, geht aus etlichen formalen sowie inhaltlichen Gesichtspunkten hervor:

\footnotetext{
15 Vgl. J. Gray, I \& II Kings. A commentary, ${ }^{3} 1980$, S. 111-112, uber die politischen Gründe, die hinter diesem Schritt stehen konnten.
} 
- Zum einen fällt er schon wegen seiner nur zwei Verse umfassenden Kürze auf.

- Von der Form her wirkt der Abschnitt auch wegen seiner strukturellen Stelle in der Mitte der Perikope literarisch hervorragend.

- $\mathrm{Zu}$ diesen formalen Betonungsaspekten kommt der inhaltliche, daß nur in Abjathars Fall nicht von einem Todesurteil die Rede ist.

- Auch das hat wiederum seine formale Seite, indem die übrigen drei Abschnitte alle regelmäßig mit einem unter dem Namen Benajas stehenden Vollzugsbericht abgeschlossen werden.

Gerade an dieser Stelle steht nun auch des Verfassers theologischer Kommentar: "So verstieß Salomo den Abjathar, daß er nicht mehr Priester Jahwes sein durfte, damit erfüllt würde das Wort Jahwes, das er in Silo über das Haus Elis gesprochen hatte".

So signalisiert der Erzähler, daß es hier um ein ganz besonderes Theologumenon geht. Seine vierte perspektivische Aussage, die er gegen Ende der Thronnachfolgegeschichte macht, unterscheidet sich von den übrigen dadurch, daß sie eine explizite theologische Geschichtsdeutung enthält.

\section{(c) Die Hauptmotive des Abjathar-Abschnittes}

Der Fall Abjathar wird abrupt, aber durchaus glaubwürdig eingeführt ${ }^{16}$, weil er, wie Joab, zur Adonia-Partei gehörte ${ }^{17}$. In den beiden Versen kommen die Motive Bestrafung, Einschränkung der Strafe und der darin implizite Vergeltungsgedanke vor:
V. 26a $\propto$ : Verbannung: Entfernung aus dem Priesterdienst.
V. 26aß.b: Todesstrafe mit Begründung deren Milderung.
V. 27: Rückblickende Notiz mit Deutung.

\footnotetext{
16 Dies ist von J.A. Montgomery \& H.S. Gehman, A critical and exegetical commentary on the Books of the Kings (ICC), Edinburgh 1951, S. 93, vom Fall Joab, nicht aber im kurzen Abjathar-Abschnitt, erkannt worden.

17 1. Kön. 1,7; 2,28; vgl. E. Würthwein, Das erste Buch der Könige. Kapitel 1-16, Göttingen ${ }^{2} 1985$, S. 23, über mögliche "religionspolitische Gründe" für die Vertreibung Abjathars; gerade das spricht eher dagegen, daß man mit Würthwein die Verse 26-27 als Nachtrag auffassen soll.
} 


\section{Das Haus Elis und das Haus Davids}

Der Abschnitt ist in zwei Teile gegliedert: V. 26 enthält, neben einer Einleitungsformel, die Worte Salomos. V. 27 enthält aber die Worte des Erzählers. Demgemäß ist die Perspektive jeweils auch unterschieden: Der König urteilt und spricht politisch, auch wenn es um religionspolitische Maßnahmen geht. Daß Salomo ihm das Leben ersparte, weil er Priester war ${ }^{18}$, ist im Licht der Bereitschaft Salomos, sogar das Heiligtum nicht zu scheuen $^{19}$, weniger wahrscheinlich. Es liegt aber in Salomos politischem Interesse, Abjathar möglichst effektiv zu isolieren. Der Erzähler hingegen urteilt und schreibt theologisch, auch wenn es ihm um die Deutung von politischen Ereignissen geht. Nach ihm verstieß Salomo den Abjathar, damit eine Prophetie über das Haus Elis erfüllt würde, womit auf 1. Sam. 2,30 zurückgegriffen wird. Abjathar, der als einziger aus der EliPriestersippe von Nob der Rache Sauls entrann ${ }^{20}$, wird eindeutig in einer berechnenden Manöverpolitik ausrangiert. Aber der Verweis auf das Haus Elis deutet das Ereignis so, daß es auf ein neues Niveau gerückt wird, nämlich das geschichtstheologische.

\section{(d) Die Prophetie gegen Eli}

In 1. Sam. 2,27-36 wird dem alten Priester ein Drohwort gegen das priesterliche Haus Elis von einem nicht näher identifizierten Propheten überbracht. Somit werden die prophetische Autorität und Wirkungskraft auch in 1. Kön. 2,27 vorausgesetzt. Ein Vergleich zwischen den beiden Texten zeigt, daß die gleichen Motive auch in 1. Sam. 2,27ff. vorkommen: Wiederum die Motive Bestrafung, Einschränkung und Vergeltung:

Vv. 30-32.34 Drohwort: Entfernung vom Priesterdienst.

Vv. 33.36 Todesmotiv und Explikation dessen Milderung.

Vv. 29.31 b Motivation und feierliche Vergeltungslehre.

\footnotetext{
18 So M. Noth, 1.Könige 1-16(BK), Neukirchen 1968, S. 35.

${ }^{19}$ Vgl. aber Ex. 21,14, wo eine Ausnahme im Asylrecht des Altars erwähnt wird, mit dem sich die Causa Joab deckt.

20 1. Sam. 22,20. Nach 1. Sam. 23,6ff. wurde Abjathar Priester des fluchtenden David und nach 2. Sam. 8,17 diente er in dieser Eigenschaft dem mittlerweile König gewordenen David gemeinsam mit einem Rivalen, Zadok. Wenn es sich um denselben Zadok handelt, hat sich dieser nach 1. Kön. 2,35 gegenüber Abjathar durchgesetzt.
} 
Der Begründung der Strafe (V. 29) zufolge, liegt die Ursache in Elis Unfähigkeit, seine Söhne zu disziplinieren: "Warum verachtet ihr meine Schlachtopfer und Speiseopfer, die ich für meine Wohnung geboten habe? Und du ehrst deine Söhne mehr als mich, daß ihr euch mästet von dem besten aller Opfer meines Volkes Israel."

Damit wird das Verhalten der Söhne Elis thematisiert (1. Sam. 2,12-17.22-25; 3,11-14). Elis Söhne hatten ein Amt im Dienst Jahwes inne, ließen sich aber nicht durch ihn stören?". Nicht nur bereicherten sie sich am Opferdienst (1. Sam. 2,13-15), sondern sie strebten ihre Ziele auch mit Gewalt an (1. Sam. 2,16) und begingen sexuelle Verbrechen (1. Sam. 2,16). Das Bild von ihrem Vater als alter, erziehungsunfähiger Mann wird plastisch geschildert: Eli vernimmt "beim ganzen volk" die Gerüchte (1. Sam. 2,23). Als Schwächling kann er nur eine weiche Ablehnung aussprechen (1. Sam. 2,2425a). Ausdrücklich wird dann die sonst völlig klare Lage vom Verfasser registriert: "Aber sie gehorchten der Stimme ihres Vaters nicht" und - aus der Erzählerperspektive mit einer theologischen Begründung motiviert: “... denn Jahwe wollte sie töten” (1. Sam. 2,25b). Abgesehen vom theologischen Problem der so suggerierten Beteiligung Gottes an einem zu bestrafenden Verbrechen ${ }^{22}$, sind die Parallelen zwischen den Personen der Eli-Erzählung und den der Geschichte von der Thronnachfolge tiefgreifend:

- David: "alt und hochbetagt"

- David: konnte nicht warm werden ${ }^{26}$; Eli: konnte nicht sehen ${ }^{27}$.

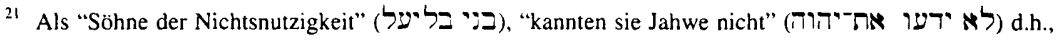
sie lebten nicht nach dem, was von einem Jahwe-Diener zu erwarten ist.

${ }^{22}$ Vgl. 2. Sam. 24,1; Ex. 4,21., 7,3.13 u.a.

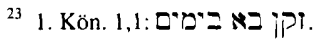

24 1. Kön. 1,15: וקאד מאר : וקר.

${ }^{25}$ 1. Sam. 2,22: מקו מאד : שאד. Vgl. 1. Sam. 4,15.

26 1. Kön. 1,1.

27 1. Sam. 4,15.
} 
- David: erfährt aus zweiter Hand, was im Volk hinsichtlich seiner Söhne passiert ${ }^{28}$; Eli: erfährt, "Reden" vom Volk über seine Söhne ${ }^{29}$.

- Davids Söhne: gewalttätig ${ }^{30}$ und sexuell ungezügelt ${ }^{31}$; Elis Söhne: drohen mit Gewalt $^{32}$ und sexuell unzüchtig ${ }^{33}$.

Diese bunte Menge von Beziehungen zwischen den Eli- und David-Erzählungen zeigt ein tiefgreifendes intertextuelles Gefüge auf. Es geht um mehr als nur ein literarisches "Abhängigkeitsverhältnis", das nur interessant ist, indem man quantitativ aufzählt, wer was von wem übernommen hat. Vielmehr geht aus dem Netzwerk hervor, daß qualitative Folgerungen aus der Wechselwirkung von Gott und Mensch in der Geschichte zu ziehen sind.

\section{(e) Die Geschichtstheologische Wirkung des Urteils über Eli}

Dem Haus Elis und dem Haus seines Vaters wurde eine immerwährende begünstigte Stellung vor Jahwe verheißen, indem dieses Geschlecht auf ewig (עדיעול ) priesterlichen Dienst verrichten sollten ${ }^{34}$. Jetzt wird these Zusage aber pauschal durch das Wort des unbekannten Propheten zurückgenommen. Die antithetische Parallelität der intensiven Konstruktion mit Infinitivus Absolutus ("ארמות) und ihres Gegensatzes (ועתה נאש-"הוחה): "Zwar hatte ich gesagt ... aber nun lautet der Gottesspruch: das sei

\footnotetext{
${ }^{28}$ 2. Sam. 13,$30 ; 15,13 ; 18,19-32 ; 1$. Kön. $1,18$.

${ }^{29}$ 1. Sam. $2,22.23$.

30 2. Sam. 13,23-37; 15,1 ff. (Absalom), 1. Kön. 2,23-25.31.46 (Salomo).

31 2. Sam. 13,1-22 (Amnon), 2. Sam. 16,22 (Absalom); in beiden Fällen weichen die Söhne nicht gerade vom väterlichen Vorbild ab (2. Sam. 11, zudem noch ein Beispiel von Davids Bereitschaft, hinterhältig Gewalt zu üben).

32 1. Sam. 2,16 .

${ }^{33}$ 1. Sam. 2,22 .

${ }^{34}$ 1. Sam 2,30a. Damit wird wahrscheinlich angespielt auf Ex 28,1. Es ist mit Montgomery \& Gehman, a.a.O., davon auszugehen, da 3 das Haus Elis und daher auch das Haus Ahitubs von Nob (1. Sam. 22,1 1) der aaronitischen Minderheitssippe der dort erwähnten Ithamariden angehörten. $\mathrm{DaB}$ der hier behauptete Gedanke des ewigen Priesterdienstes nicht spezifisch mit dem Ithamar-Geschlecht in Verbindung gebracht wurde, schwächt die Wirkungskraft des Motivs in 1. Sam. 2 keineswegs, sondern ist eher eine Verstärkung, weil der tendenziöse Charakter des Motivs in der Eli-Erzählung so noch deutlicher wird.
} 
ferne von mir!". Damit wird die Frage des Jonabuches - ob ein einmal gegebenes Gotteswort zurückgenommen werden kann - eindeutig positiv beantwortet. Während etwa die Jona-Erzählung und Abrahams Eintreten für Sodom (Gen. 18,16-33) das Rückgängigmachen einer Drohung verteidigen, wird das Zurücknehmen einer Verheißung in unserem Text expliziert.

An sich hätte das schon erhebliche theologische Bedeutung, aber, weil dieses Theologumenon in einem intertextuellen geschichtstheologischen Netzwerk aufgenommen ist, wird es im Blick auf ein weiteres Theologumenon konkretisiert. Das Gültigbleiben einer göttlichen Verheißung wird nämlich mit der menschlichen Verantwortung verbunden. In V. $30 \mathrm{~b}$ heißt es feierlich im antithetischen Parallelismus:

\section{"Denn meine Verehrer werde ich ehren} und meine Verächter werden verachtet werden."

Hier wird mit Vergeltung nach der Art der lex talionis gerechnet. ' ("denn") gibt den Grund an. Weil das Haus Elis sich im amtlichen Dienst versündigt hat, wird es trotz der Verheißung verworfen. Daran sind die Söhne Hofni und Pinhas schuld, aber ebensoviel Eli, weil seine fehlende Erziehungsfähigkeit den Söhnen mehr Ehre als Jahwe zukommen ließ und weil das Talionsprinzip sich auch auf die Strafe erstreckt: Wenn eine ewige Verheißung zurückgenommen wird, kommt ein ewiges Gericht an ihrer Stelle ${ }^{35}$. Das Vergeltungs- oder Talionsprinzip scheint mir hier nicht an sich oder unabhängig zu funktionieren, sondern als unterstützende Begründung für den Hauptgedanken, nämlich das aufgehobene Gotteswort. Damit wird die Verantwortungskategorie eingeführt: Weil Jahwe das menschliche Verhalten ins Kalkül bringt, darum kann er seine früher verabschiedeten Ankündigungen rückgängig machen. Der Mensch ist also mitbestimmend in der Geschichte wirksam. Zwar bleibt Jahwe der Überlegene, der die Initiative ergreifen kann und es auch tut (wie es eben in dieser Erzählung zu beobachten ist), aber er nimmt das Benehmen des Menschen durchaus ernst.

\footnotetext{
35 1. Sam. 2,29; 3,13. Im letzteren Text wird die ewige Priesterschaft mit einem ewigen Gericht bilanziert.
} 
Mit seiner Rücksichtnahme auf die Eli-Erzählung signalisiert der Verfasser der Geschichte von der Thronnachfolge nicht nur seine Perspektive in dem Sinne, daß er uns verrät, wie er die Geschichte versteht. Vielmehr weist der oben angedeutete feierlichformale Charakter der Erfüllungserklärung darauf hin, daß es sich um etwas Grundsätzliches handelt, also um etwas, das programmatisch für die Zukunft zu beachten ist.

Hierin liegt zutiefst beunruhigender Kommentar. Gerade in dem Kapitel der Thronfolgegeschichte, wo dieser Hinweis steht, kommen wiederholte Verweise auf den immerwährenden Bestand des Davidshauses vor ${ }^{36}$. Auch das wird aber als bedingte Möglichkeit formuliert ${ }^{37}$. Damit wird eine weitere intertextuelle Beziehung aufgerufen: die Nathansverheißung in 2. Sam. 7,13: "(Dein Nachkomme) soll meinem Namen ein Haus bauen, und ich werde den Thron seines Königtums ewiglich bestätigen". Der literarische Effekt ist nicht zu überhören: Jeder, der die Geschichte von Eli und seiner Familie aus der Zeit Samuels kennt, wird erinnert daran, was passiert, wenn ein alter Mann seine Kinder nicht disziplinieren kann, wenn die Gebote Jahwes nicht eingehalten werden, wenn Gewalt losbricht: Die Zusage Gottes wird zurückgenommen - es folgt Verwerfung.

Die Absetzung des Eliden, Abjathar, durch Salomo ist also der Nachweis, daß er selbst und die Dynastie zur Verantwortung gerufen werden. Wenn Jahwes Zusicherung an das Haus Elis zu einem Ende kommen konnte, dann kann auch seine Zusicherung an das Haus Davids zu einem Ende kommen. Das Schlußwort der Thronfolge-Erzählung, daß das Königtum in Salomos Hand gefestigt war, wird also durch diese dunklen, kontrapunktischen Töne gründlich relativiert. Von der Perspektive des Erzählers aus heißt dies die Konstruktion einer "pro-dynastischen" Linie bis zu den letzten Worten der Geschichte, sowie eine de-Konstruktion derselben durch den unterminierenden Effekt des “anti-dynastischen" Elements. Diese Dekonstruktion ist laufend durch die Thronnachfolge-Erzälung hindurch mit der Eli-artigen Charakterisierung Davids und mit der

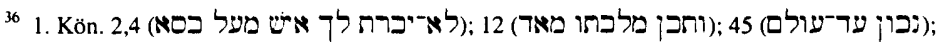
46 (כבונה)

37 1. Kön. 1,4, wo die Eא-Konstruktion die Nachfolger Davids am Gesetz des deuteronomistisch klingenden 3 . Verses bindet.
} 
Charakterisierung seiner Söhne nach Art des Hophni-Phinehas-Bildes vorbereitet worden. Nicht von ungefähr steht der Abjathar-Eli-Hinweis in der Mitte des Kapitels, in dem Salomo, ebenso gewaltsam, wenn nicht gewaltsamer als die Söhne Elis erscheint. Nicht nur die gescheiterten Beansprucher des Thrones, Absalom, Amnon und Adonia, sind in mancher Hinsicht Ebenbilder ihres Vaters, sondern auch der erfolgreiche Salomo ähnelt ihm. Das frühe Porträt des neuen Königs stellt die beruhigende Schlußaussage der Thronfolgegeschichte in Frage: Wie fest hat der Mann, der den Söhnen Elis nicht unähnlich ist und dessen Vater dem Bild ihres Vaters nicht unähnlich ist, den Thron wirklich “in seiner Hand"?

\section{THEOLOGISCHE ENTWICKLUNGSMÖGLICHKEITEN}

Wir haben oben gesehen, daß die von Von Rad identifizierten Deutungen des Erzählers sowohl Kritik am davidischen Thron als auch Hinweise auf die freie Liebe Gottes für ihn enthalten. Das berechtigt schon, von einer kritischen sowie einer positive Seite der Erzählungstendenz zu sprechen. Gott hat die frühen Schicksale des Davidshauses in günstige Wege gelenkt, aber es ist durchaus möglich, daß er das Haus mangels verantwortungsvollen Auftretens wieder verwirft.

- Versuchen wir, dieses Fazit unserer Untersuchung in einen breiteren Rahmen zu legen, müssen die Kategorien, in denen die beiden Aspekte der Erzählungstendenz untergebracht werden sollen, das Doppelthema sein, das die kritischen Propheten ebenso wie das deuteronomistische Korpus prägt: Einerseits die freie Liebe Gottes und andererseits seine Forderung des Gehorsams. In dieser Erzählung gilt das an erster Stelle für die Dynastie, ist aber als solches die Manifestation eines Grundsatzes. Daher können diese beiden Kategorien auch über die Grenze der Thronfolgegeschichte hinaus im übergreifenden Deuteronomistischen Werk als geschichtstheologisches Kriterium für die Deutung von Israels Schicksal verfolgt werden. Hier funktionieren sie als geschichtstheologisches Kriterium für die Deutung der Geschichte bis zum Exil, also aus der Perspektive des Exils. Zwar sind die Könige die primären Objekte der Beurteilung, aber das Kriterium wird auch für das ebenso vom Schicksal betroffene Volk 
verwendet. Die ganze Geschichte Israels wird im Spannungsfeld der Aufeinanderbezogenheit dieser Pole interpretiert. ${ }^{38}$

- Diese beiden Aspekte kommen auch in dem von Spieckermann" "die Gnadenformel" genannte Bekenntnis von Ex. 34,6 und Num. 14,18 vor $^{40}$. Nach Spieckermann wird "Jahwe inmitten von Eigenschaften präsentiert, deren Auswahl sein Wesen offensichtlich möglichst umfassend beschreiben soll". Das stimmt, aber aufs engste mit dieser "Gnadenformel" verbunden ist die Tatsache, daß Jahwe die Sünde trotzdem nicht ungestraft läßt ${ }^{4 l}$. Liebe und Gnade, ebenso aber Gerechtigkeit und Gehorsamsforderung sind die Aspekte des göttlichen Wesens. Die erste Seite bietet Raum für den positive Aspekt, und die zweite für die kritische Evaluierung der Dynastie in der Thronfolgegeschichte sowie im Deuteronomistischen Geschichtswerk als Ganzes.

- Spieckermann thematisiert die Möglichkeit, in der "Gnadenformel" die Mitte des Alten Testaments zu suchen. Dazu möchte ich fragend hinzufügen: Wenn eine Mitte des Alten Testaments doch aus der Perspektive einer durchdachten Komposition möglich wäre, wäre es nicht auch denkbar, von der Wechselwirkung zwischen den beiden Seiten des Bekenntnisses aus bzw. von der Dynamik der beiden Aspekte dieses Geschichtskriteriums aus zu einem theologischen Ordnungsprinzip zu kommen, das nicht nur die Vielfalt in der Einheit, sondem auch die Spannungen respektiert? Auch die Fraktion von Beziehungen zwischen Klein- und Großerzählungen, Intertexten und Büchern, die wir in diesem Netz

\footnotetext{
${ }^{38}$ Es scheint mir, daß die Schwäche von Von Rads oben erwähnten Sicht gerade hier liegt. Er scheint dem positiven Aspekt alle Beachtung zu schenken, den von ihm gut erkannten kritischen Aspekt aber zu vernachlässigen, indem er ihn als Objektivität des Erzählers mißversteht. So kann seine Auffassung des Anliegens der Königsbücher als das Funktionieren des göttlichen Wortes in der Geschichte zwar nicht als falsch, wohl aber als einseitig heilsgeschichtlich betrachtet werden.

${ }^{39}$ H. Spieckermann, "Barmherzig und gnădig ist der Herr ..." ZAW 102 (1990), 1-1 8.

40 Vgl. auch Joel 2,13; Jona 4,2; Ps. 86,15;103,8; 145,8; Neh. 9,17. Dazu Spieckermann a.a.O. (der Num.14:18 nicht zu den Hauptbelegen zählt).

41 Vgl. Ex. 34,6 plus 7; Num. 14,18a plus b.
} 
untersucht haben, scheint mir auf diese Potenz zu deuten. Nicht nur die alttestamentliche Theologie, sondern vor allem die heute neu zu stellende Gottesfrage brauchen Kriterien um über den alle Kriterien sprengenden Gott zu reden. Was er gesagt hat, muß nicht passieren. Was er nicht gesagt hat, kann passieren. Denn er ist Gott. 